\title{
Extensively Drug-Resistant (XDR) Tuberculosis: A Review
}

\author{
AHMED JU ${ }^{\mathrm{a}}$, MUSA AKM ${ }^{\mathrm{c}}$, HOSSAIN $\mathrm{D}^{\mathrm{b}}$, RAHIM MA ${ }^{\mathrm{a}}$, SHAHEEN AKM ${ }^{\mathrm{b}}$, UDDIN KN ${ }^{\mathrm{d}}$
}

\begin{abstract}
:
Extensively drug-resistant (XDR) tuberculosis is defined as disease caused by Mycobacterium tuberculosis with resistance to at least isoniazid and rifampicin, any fluoroquinolone, and at least one of three injectable secondline drugs (amikacin, capreomycin, or kanamycin). This definition in immensely valuable for more uniform surveillance in varied international settings. The prevalence of tuberculosis drug resistance has risen to the highest rate ever recorded. Although the gold standard for drugsusceptibility testing has been the agar proportion method; due to it's time consumption, more sensitive, specific and rapid diagnostic tests are required. It is difficult to differentiate XDR tuberculosis from non-XDR tuberculosis
\end{abstract}

\section{Introduction:}

Extensively drug-resistant (XDR) tuberculosis has received substantial attention since the initial report of an association of XDR-TB with extremely high mortality in patients co-infected with $M$. tuberculosis and HIV in a rural area of South Africa in the year 2006. ${ }^{1}$ XDR strains of $M$. tuberculosis have now been identified in at least 49 countries around the globe. ${ }^{2}$

From the advent of tuberculosis chemotherapy in the 1940s, hints of resistance were evident. When Selman Waksman accepted the Nobel Prize in 1952 for his laboratory's discovery of streptomycin, he claimed the drug would lead the path to the elimination of "The Great White Plague".3, 4 Such statements were premature strains of streptomycin-resistant Mycobacterium tuberculosis were found within months of the drug's widespread use. ${ }^{5}$ The classic 1948 British Medical

a. Ahmed JU, Rahim MA, Registrar, Department of Internal Medicine \& Pulmonology, BIRDEM

b. Hossain D, Shaheen AKM, Assistant Professor, Department of Internal Medicine \& Pulmonology, BIRDEM

c. Musa AKM, Associate Professor, Department of Internal Medicine \& Pulmonology, BIRDEM

d. Uddin KN, Professor, Department of Internal Medicine \& Pulmonology, BIRDEM

Address of Correspondence: Dr. Jamal Uddin Ahmed, MBBS, FCPS (Medicine), Registrar, Department of Internal Medicine \& Pulmonology, BIRDEM, Room No. 1537, 14 ${ }^{\text {th }}$ Floor, BIREDM Hospital, Phone: 01671064214, Email: jmldollar@ gmail.com Received: May 30, 2011 Accepted: June 30, 2011 clinically, although the former is associated with greater morbidity and mortality. The treatment of XDR tuberculosis should include agents to which the organism is susceptible, and should continue for a minimum of 18-24 months. However, treatment continues to be limited in tuberculosisendemic countries largely because of weaknesses in national tuberculosis health-care models. The ultimate strategy to control drug-resistant tuberculosis is one that implements a comprehensive approach incorporating innovation from the political, social, economic, and scientific realms.

Key words: Extensively drug-resistant (XDR) tuberculosis, Mycobacterium tuberculosis

(Birdem Med J 2011; 1(1): 30-36)

Research Council (BMRC) trial that investigated the efficacy of streptomycin monotherapy showed that most patients who were treated with the drug developed resistant strains. ${ }^{6}$

As the tuberculosis chemotherapy era evolved, increasing cases of drug resistance continued to occur mainly as a result of inadequate regimens and nonadherence to therapy. Researchers initially suspected that these resistant organisms had reduced fitness and thus could be classified as being less virulent. ${ }^{7,8}$ This assumption was reversed in the 1990s with the rise in multidrug-resistant (MDR) tuberculosis-ie, M tuberculosis resistant to at least rifampicin plus isoniazid. ${ }^{9}$ Substantial attention was focused upon New York City (NY, USA) where a virulent and transmissible strain had spread among immunocompromised populations. ${ }^{10,11}$ Awareness of tuberculosis drug resistance was refocused with a study presented in August, 2006, at the XVI International AIDS Conference in Toronto, Canada, which described an epidemic of XDR tuberculosis in a rural hospital in KwaZulu-Natal Province, South Africa. ${ }^{12}$

\section{Definition of XDR tuberculosis:}

The term XDR tuberculosis was first developed by the US Centers for Disease Control and Prevention (CDC) in March, 2005. ${ }^{13}$ It came to public focus in October, 2005, at the 36th Union World Conference on Lung Health in Paris, France. ${ }^{14,15}$ The original definition 
was proposed in March, 2006, in CDC's Morbidity and Mortality Weekly Report, defining it as M tuberculosis with resistance to at least isoniazid and rifampicin among the first-line tuberculosis drugs and resistance to at least three of the six main classes of second-line drugs (aminoglycosides, polypeptides, fluoroquinolones, thioamides, cycloserine, and aminosalicylic acid). ${ }^{16}$ This definition was subsequently revised in October, 2006, during the first meeting of the WHO Global XDR-TB Task Force. The classification, which continues to be accepted, requires resistance of $\mathrm{M}$ tuberculosis to at least isoniazid and rifampicin, any fluoroquinolone, and at least one of three injectable second-line drugs (capreomycin, kanamycin or amikacin). ${ }^{17}$ The revision was made to facilitate reproducibility of drugsusceptibility testing and to focus attention on drugs accessible in resource-limited settings. Moreover, the classification has also been shown to have value in its ability to predict poorer outcomes. ${ }^{18}$

\section{Epidemiology:}

In the face of rising cases of MDR tuberculosis, WHO and the International Union Against Tuberculosis and Lung Diseases (IUATLD) established the Global Project on Anti-tuberculosis Drug Resistance Surveillance in the early 1990s. ${ }^{19}$ One of the most important outcomes of the project was the formation of an international quality assurance programme supervised by supranational reference laboratories (SRLs). ${ }^{20}$ There are currently 26 SRLs that assist over 100 national laboratories in six continents by standardizing culture and drug-susceptibility techniques. To determine the rate of XDR tuberculosis, CDC and WHO assessed 17,690 $\mathrm{M}$ tuberculosis isolates collected by 25 SRLs from 2000 -2004. ${ }^{16}$ The study found that $20 \%$ of the isolates met MDR-tuberculosis criteria and $2 \%$ were classifiable as XDR tuberculosis. Population-based assessment showed that $4 \%, 15 \%$, and $19 \%$ of XDR-tuberculosis cases were obtained from the USA, South Korea, and Latvia, respectively. ${ }^{16}$

In a rural hospital in Tugela Ferry, KwaZulu-Natal Province, South Africa, 1539 individuals were tested for tuberculosis from January, 2005, to March, 2006, 542 had at least one culture that was positive for $\mathrm{M}$ tuberculosis. Of these 542 patients with confirmed tuberculosis, 53 had XDR tuberculosis. ${ }^{21}$ Factors that have fuelled this South African epidemic include ineffective tuberculosis treatment in the context of a high prevalence of HIV, lack of proper diagnostic testing, and poor infection control practices. ${ }^{22,23}$

The occurrence of MDR tuberculosis has reached its highest level, with cases reported in a record 49 countries. In South Africa, Tomsk Oblast (Russian Federation), and Estonia-all countries with a high burden of tuberculosis- $5 \cdot 7 \%, 6 \cdot 6 \%$, and $23 \cdot 7 \%$ of all MDR-tuberculosis cases were XDR, respectively.

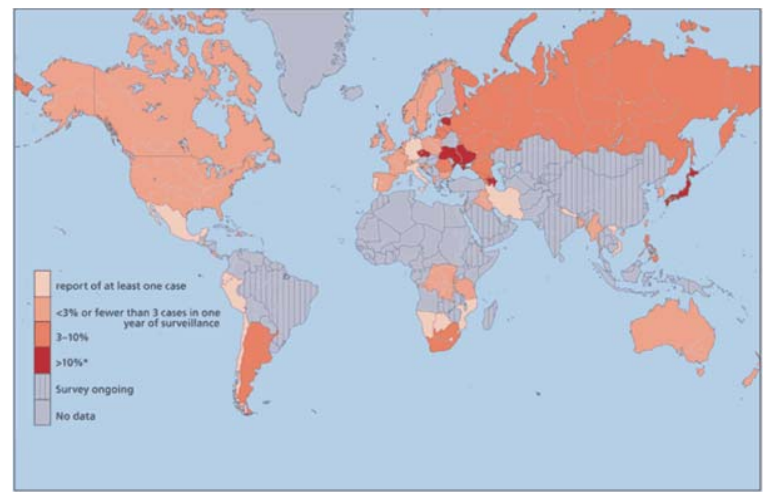

The USA, a country with a low tuberculosis prevalence, recently reclassified $1.9 \%$ of MDR-tuberculosis cases as XDR tuberculosis. ${ }^{3}$

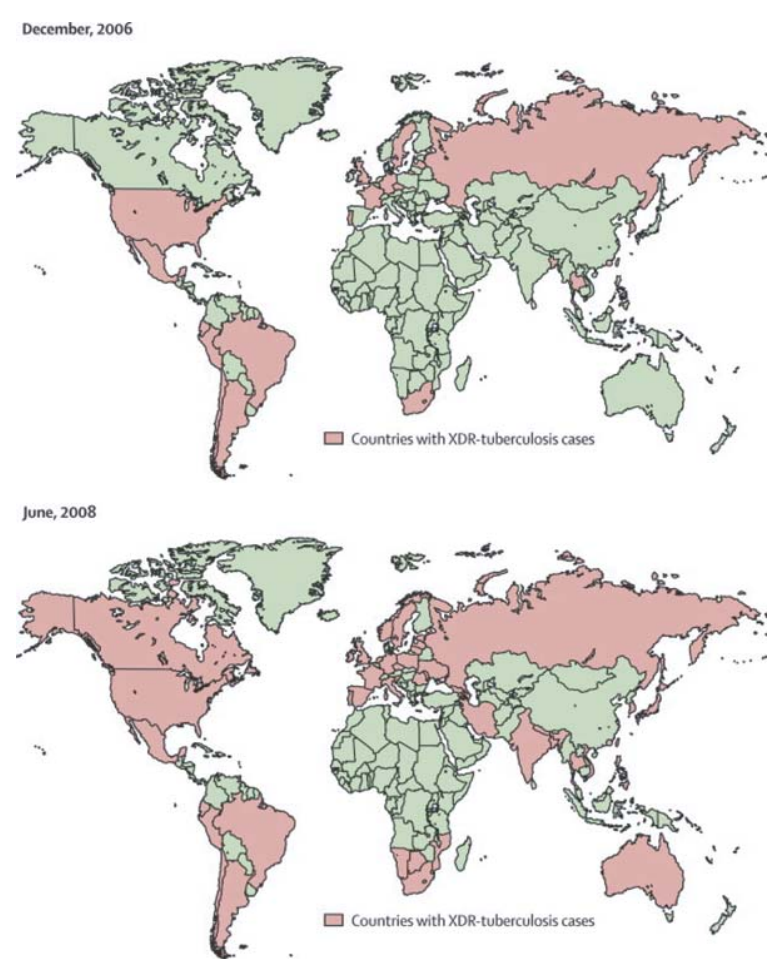

Figure: Countries with XDR-tuberculosis cases in December, 2006, and June, 2008 
According to ICDDRB report, MDR surveillance is continuing at Shyamoli TB clinic, Dhaka, Bangladesh in collaboration with National TB control programme (NTP). In their observation, out of 657 isolates, multidrug resistance was observed in 5.5\% isolates. It was significantly higher among persons who received tuberculosis treatment for e" one month. (15.4\% vs $3.0 \%)$

\section{Mechanisms of resistance and fitness in XDR tuberculosis:}

The basis of tuberculosis drug resistance is the selection of bacterial mutants with innate resistance to chemotherapy. ${ }^{24,} 25$ Epidemics of drug-resistant disease can be generated by three interrelated mechanisms: (1) conversion of wildtype pan-susceptible strains to drugresistant strains during treatment (acquired resistance); (2) increasing development of resistance in drugresistant strains because of inappropriate chemotherapy (amplified resistance); and (3) transmission of drugresistant cases (transmitted resistance). ${ }^{26}$

Acquired and amplified drug resistances are the primary means by which tuberculosis drug-resistant strains have been generated. However, the key determinant that has led to the exponential rise in XDR-tuberculosis cases is likely to have been transmitted resistance. The role of transmitted resistance can be elucidated by noting the clonal strains evident in tuberculosis outbreaks. The MDR-tuberculosis outbreak in the early 1990s in New York City, fuelled by the HIV epidemic and urban settings, was primarily associated with a clinically virulent strain of Beijing/W genotype. ${ }^{27}$ Moreover, 39 (85\%) of 46 isolated XDR-tuberculosis strains in the Tugela Ferry outbreak in South Africa belonged to the KwaZulu-Natal (KZN) genotypic family of strains. ${ }^{21}$ The transmission of drug-resistant tuberculosis largely depends on the virulence of the mutated organism. ${ }^{28-30}$

\section{Diagnostic tests for XDR tuberculosis:}

The gold standard for drug-susceptibility testing has been the agar proportion method on Lowenstein-Jensen medium and Middlebrook 7H11 agar. ${ }^{31}$ But, there are several disadvantages. The reproducibility and accuracy of drug-susceptibility testing for second-line antituberculosis drugs remains questionable. The technique can take up to $4-8$ weeks for finalized results. Thus, the inability to detect drug resistance rapidly could increase the likelihood of an isolate developing resistance through ineffective chemotherapy, ${ }^{32}$ likelihood of transmission of resistant strains and the potential to produce clusters of secondary infections. ${ }^{33}$ Line-probe hybridization assays in conjunction with nucleic-acid amplification offer a promising route to rapid identification of isoniazid and rifampicin resistance and are currently being studied by the WHO SRLs. ${ }^{35}$

Other molecular methods that could be used in the detection of drug-resistant tuberculosis strains ${ }^{34}$ include the molecular beacon assay, luciferase mycobacteriophage strategy, dideoxy fingerprinting, direct sequencing of PCR products, and heteroduplex analysis. These methods are generally described as providing results rapidly and being highly sensitive. However, they are also labour intensive and costly compared with the agar proportion method.

One particular form of testing that has received much attention because of its potential application in resourcelimited settings is the microscopic-observation drugsusceptibility (MODS) assay. A median time of only 7 days (IQR 6-8 days) is needed for both disease identification and drug-susceptibility testing. ${ }^{36}$

\section{Clinical course of XDR tuberculosis:}

MDR tuberculosis is associated with a high mortality in individuals with HIV or other immunosuppressive conditions. ${ }^{37}$ The even poorer clinical outcomes associated with XDR tuberculosis was initially documented in the first CDC report of the disease in 2006. ${ }^{13}$ During 1993-2002, patients with XDR tuberculosis were $64 \%$ more likely to die during treatment than patients with MDR tuberculosis. ${ }^{10,13}$

An appreciation for the substantial morbidity and mortality associated with co-infection with XDR tuberculosis and HIV was heightened by the findings in KwaZulu-Natal. ${ }^{21}$ Fifty two (98\%) of 53 patients with XDR tuberculosis died during the study period. Forty four (83\%) of the 53 XDR-tuberculosis patients agreed to HIV testing and all tested positive for co-infection.

The first published case reports of XDR tuberculosis in India noted that among 54 HIV-positive patients, 4 (33\%) of 12 diagnosed MDR tuberculosis cases were reclassified as XDR tuberculosis after drugsusceptibility testing. ${ }^{37}$ All the patients with XDR tuberculosis died within 2-6 months of diagnosis. A recent study from South Korea described the clinical 
outcomes of 43 HIV-uninfected patients with XDR tuberculosis. ${ }^{38}$ Treatment failure, defined as a lack of culture conversion, was noted in 19 (44\%) patients with XDR tuberculosis compared with 46 (27\%) non-XDRtuberculosis patients. Moreover, the mortality was $14 \%$ in those with XDR tuberculosis and 8\% in those with MDR tuberculosis. A five-fold increase in the risk of death in patients with XDR tuberculosis was seen in a study done in Germany and Italy. ${ }^{39}$ XDR-tuberculosis patients required longer hospital stays and longer treatment durations, mainly because of clinical complications (ie, sputum conversion).

\section{Treatment of XDR tuberculosis:}

The principles of treatment for MDR-TB and for XDRTB are the same. Treatment requires extensive chemotherapy for up to two years. Second-line drugs are more toxic than the standard anti-TB regimen and can cause a range of serious side-effects including hepatitis, depression and hallucinations. Patients are often hospitalised for longer periods, in isolation. In addition, second-line drugs are extremely expensive compared with the cost of drugs for standard TB treatment.

Strategies to treat drug-resistant tuberculosis can be categorized as either standardized or individualised. ${ }^{40}$ Standardized regimens are determined on representative drug-resistance surveillance data of specific regions. Individualized regimens are more specific in that they take into account previous antituberculosis treatments and drug-susceptibility testing of the particular isolate. XDR tuberculosis requires individualized treatment given the inability of standardized regimens to accurately address both first-line and second-line treatment resistance. ${ }^{41,42}$ Individualized regimens are also the only reliable means by which the amplification of drug resistance may be avoided. ${ }^{43}$ Unfortunately, the difficulty in performing drug-susceptibility testing in many resource-limited countries has led to long-term use of inadequate empiric regimens that could lead to further acquired resistance. ${ }^{44}$

The length of treatment for XDR tuberculosis has not been firmly established and is often based on individual clinical presentations. ${ }^{45}$ Key factors determining treatment duration include cost, drug availability, toxicity, bactericidal capacity, clinical improvement, and patient adherence. ${ }^{41}$ Typical MDR-tuberculosis regimens can consist of up to five drugs, and WHO recommends their use for a minimum of 18 months of treatment after culture conversion to negative 46,47 Treatment of XDR tuberculosis should include agents that the strain of $M$ tuberculosis has proven to be susceptible to. Any first-line agent to which the isolate has shown to be susceptible, and any appropriate second-line drugs should be used to achieve a regimen with a minimum of four to five effective medications. ${ }^{48}$ Treatment with this regimen should be continued for a minimum of 18 - 24 months.

Grouping of anti-tubercular drugs:

\begin{tabular}{ll}
\hline Grouping & Drugs (Abbreviation) \\
\hline $\begin{array}{l}\text { Group 1- First-line oral } \\
\text { antituberculosis agents }\end{array}$ & $\begin{array}{l}\text { Isoniazid (H); Rifampicin (R); Ethambutol (E); } \\
\text { Pyrazinamide (Z) }\end{array}$ \\
$\begin{array}{l}\text { Group 2 - Injectable } \\
\text { antituberculosis agents }\end{array}$ & Streptomycin (S); Kanamycin (Km); Amikacin \\
Group 3 & (Am); Capreomycin (Cm); Viomycin (Vi) \\
Fluoroquinolones & Ciprofloxacin (Cfx); Ofloxacin (Ofx); Levofloxacin \\
Group 4 -Oral bacteriostatic & (Lfx); Moxifloxacin (Mfx);e Gatifloxacin (Gfx) \\
second-line antituberculosis & Ethionamide (Eto); Protionamide (Pto); \\
agents & Cycloserine (Cs); Terizidone (Trd) ; Raminosalicylic \\
$\begin{array}{l}\text { Group 5 -Antituberculosis agents } \\
\text { efficacy (not recom-mended by }\end{array}$ & acid (PAS); Thioacetazone (Th)h \\
$\begin{array}{l}\text { WHO for routine use } \\
\text { in MDR TI3 patients) }\end{array}$ & Clofazimine (Cfz); Amoxicillin/Clavulanate (Amx/ with unclear \\
\hline
\end{tabular}


A possible treatment regimen is as follows- The treatment is divided into 2 phages: An initial phase of 6 months, which is extended to 9 months if the sputum culture is positive at $4^{\text {th }}$ month; to be followed by a continuation phase of minimum 18 months. Sputum smear examination should be conducted monthly during initial phase and quarterly during continuation phase. Sputum culture should be done at least at 4, 6, 12, 18, $24^{\text {th }}$ month.

A study in the Tomsk oblast of Russia, reported that 14 out of 29 (48.3\%) patients with XDR-TB successfully completed treatment. ${ }^{49}$ In Hong Kong the overall treatment success rate of XDR tuberculosis compared to MDR tuberculosis is $38 \%$ vs $63 \%$.

Surgical treatment should also be considered if clinically significant parenchymal lung disease is localized and high-grade resistance is present. ${ }^{50}$ Bilateral disease can also be approached surgically but requires multiple, staged resections. Cure rates of MDR tuberculosis can be greater than $90 \%$ with post-surgical chemotherapy. ${ }^{51}$

In view of the multiple drug cross-resistance patterns, new antituberculosis drugs with novel mechanisms of action are necessary if XDR tuberculosis is to be successfully treated. Future treatment also requires development of drugs with minimal adverse events. Ideally, such agents would not have pharmacological interactions with antiretroviral drugs commonly used to treat HIV. Promising new compounds with high potency against $\mathrm{M}$ tuberculosis include a diarylquinoline compound (R207910, also called TMC207) and two nitroimidazole compounds (PA-824 and OPC67683). ${ }^{52-54}$ Moreover, tuberculosis vaccines are currently being tested which might serve as immunotherapeutic agents to accompany tuberculosis drug regimens. ${ }^{55}$

\section{Prevention:}

A multifaceted approach is advocated to address the XDR-tuberculosis epidemic. The WHO Global XDRTB Task Force initially established comprehensive recommendations in 2006 after recognizing the impact of the disease. ${ }^{56}$

Recommendations of the WHO Global Task Force on XDR-TB, Oct 9, 2006.

- Improve global tuberculosis control by enhancing the testing and care of HIV-infected populations
- Develop programme management and treatment guidelines of XDR tuberculosis in high and low HIV prevalence settings

- $\quad$ Strengthen laboratory diagnostic services to ensure rapid and accurate drug-susceptibility testing

- $\quad$ Reducing transmission in health-care settings and other high-risk areas to improve infection control

- Increase disease surveillance efforts to accurately assess epidemiological trends

- Enhance educational advocacy and research funding to encourage development of new drugs and diagnostics

- Improve global tuberculosis control by enhancing the testing and care of HIV-infected populations

\section{Conclusion:}

The rising prevalence of XDR tuberculosis has brought a resurgence of interest in drug-resistant tuberculosis. Because of a confluence of several epidemiological factors - such as the HIV pandemic and inadequate case detection and treatment completion-virulent XDRtuberculosis strains have been increasingly reported worldwide. The development of highly sensitive and rapid laboratory tests for tuberculosis diagnosis also remains an area worthy of further investigative efforts. Immediate action can be implemented through the use of currently available strategies such as enhanced HIV detection and treatment, improved tuberculosis diagnostics (ie, MODS and line-probe hybridisation assays), effective infection control policies (ie, isolation, natural ventilation, and respiratory masks), and increasing local advocacy/research efforts

\section{References:}

1. Marris E. Extreme TB strain threatens HIV victims worldwide. Nature 2006; 443: 131- 34.

2. Baleta A. Forced isolation of tuberculosis patients in South Africa. Lancet Infect Dis 2007; 7: 771-75.

3. Shatz A. Effect of streptomycin and other antibiotic substances upon Mycobacterium tuberculosis and related organisms. Proc Soc Exp Biol Med 1944; 57: 244-48.

4. Nobel Foundation. Selman A Waksman-from Nobel lecture, physiology or medicine 1942-1962. Amsterdam: Elsevier Publishing Company, 1964.

5. Cannetti G. The J Burns Amberson lecture: present aspects of bacterial resistance in tuberculosis. Am Rev Respir Dis 1965; 92: 687-703.

6. British Medical Research Council. Streptomycin treatment of pulmonary tuberculosis. BMJ 1948; 2: 769-82. 
7. Gillespie SH. Tuberculosis: evolution in millennia and minutes. Biochem Soc Trans 2007; 35: 1317-20.

8. Gillespie SH, Billington OJ, Breathnach A, McHugh TD. Multiple drug-resistant Mycobacterium tuberculosis: evidence for changing fitness following passage through human hosts. Microb Drug Resist 2002; 8: 273-79.

9. CDC. Nosocomial transmission of multidrug-resistant tuberculosis to health-care workers and HIV-infected patients in an urban hospital-Florida. MMWR Morb Mortal Wkly Rep 1990; 39: 718-22.

10. Frieden TR, Sterling T, Pablos-Mendez A, Kilburn JO, Cauthen GM, Dooley SW. The emergence of drug-resistant tuberculosis in New York City. N Engl J Med 1993; 328: 521-26.

11. Frieden TR, Fujiwara PI, Washko RM, Hamburg MA. Tuberculosis in New York City. N Engl J Med 1995; 333: 229-33.

12. Gandhi NR, Moll A, Pawinski R. High prevalence and mortality from extensively-drug resistant (XDR) TB in TB/ HIV coinfected patients in rural South Africa. XVI International AIDS Conference; Toronto, Canada; Aug 1318, 2006. Abstract THLB0210.

13. Holtz TH. XDR-TB in South Africa: revised definition. PLoS Med 2007; 4: 161-64.

14. Shah NS, Wright A, Drobniewski F. Extreme drug resistance in tuberculosis (XDR-TB): global survey of supranational reference laboratories for Mycobacterium tuberculosis with resistance to second-line drugs. Int J Tuberc Lung Dis 2005; 9 (suppl 1): 77-83.

15. Holtz TH, Riektsina V, Zarovska E, Laserson KF, Wells CD, Leimane V. Extreme drug-resistance and treatment outcome under DOTS-Plus, Latvia, 2000-2002. Int J Tuberc Lung Dis 2005; 9 (suppl 1): 258-62.

16. CDC. Emergence of Mycobacterium tuberculosis with extensive resistance to second-line drugs-worldwide, 20002004. MMWR Morb Mortal Wkly Rep 2006; 55: 301-05.

17. CDC. Extensively drug-resistant tuberculosis—United States, 1993-2006. MMWR Morb Mortal Wkly Rep 2007; 56: 25053.

18. Raviglione MC, Smith IM. XDR tuberculosis-implications for global health. N Engl J Med 2007; 356: 656-59.

19. Cohn DL, Bustreo F, Raviglione MC. Drug resistance in tuberculosis: review of the worldwide situation and WHO/ IUATLD's Global Surveillance Project. Clin Infect Dis 1997; 24 (suppl 1): 121-30.

20. Laszlo A, Rahman M, Espinal M, Raviglione M. Quality assurance program for drug susceptibility testing of Mycobacterium tuberculosis in the WHO/IUATLD Supranational Reference Laboratory Network: five rounds of proficiency testing, 1994-1998. Int J Tuberc Lung Dis 2002; 6: 748-56.
21. Gandhi NR, Moll A, Sturm AW. Extensively drug-resistant tuberculosis as a cause of death in patients co-infected with tuberculosis and HIV in a rural area of South Africa. Lancet 2006; 368: 1575-80.

22. Raviglione MC, Uplekar MW. WHO’s new Stop TB Strategy. Lancet 2006; 367: 952-55.

23. Dowdy DW, Chaisson RE, Moulton LH, Dorman SE. The potential impact of enhanced diagnostic techniques for tuberculosis driven by HIV: a mathematic model. AIDS 2006; 20: 751-62.

24. Gillespie SH. Evolution of drug resistance in Mycobacterium tuberculosis: clinical and molecular perspective. Antimicrob Agents Chemother 2002; 46: 267-74.

25. Dorman SE, Chaisson RE. From magic bullets back to the Magic Mountain: the rise of extensively drug-resistant tuberculosis. Nat Med 2007; 13: 295-98.

26. Blower SM, Chou T. Modeling the emergence of the "hot zones": tuberculosis and the amplification dynamics of drug resistance. Nat Med 2004; 10: 1111-16.

27. Frieden TR, Woodley CL, Crawford JT, Lew D, Dooley SM. The molecular epidemiology of tuberculosis in New York City: the importance of nosocomial transmission and laboratory error. Tuber Lung Dis 1996; 5: 407-13.

28. Blower SM, Gerberding JL. Understanding, predicting and controlling the emergence of drug-resistant tuberculosis: a theoretical framework. J Mol Med 1998; 76: 624-36.

29. Dye C, Williams BG. Criteria for the control of drug-resistant tuberculosis. Proc Natl Acad Sci USA 2000; 97: 8180-85.

30. Dye C, Espinal MA. Will tuberculosis become resistant to all antibiotics? Proc Biol Sci 2001; 268: 45-52.

31. Brodie D, Schuluger NW. The diagnosis of tuberculosis. Clin Chest Med 2005; 26: 247-71.

32. Farmer P, Bayona J, Becerra M. The dilemma of MDR-TB in the global era. Int J Tuberc Lung Dis 1998; 2: 869-76.

33. Espinal MA. Time to abandon the standard treatment regimen with first-line drugs for failures of standard treatment. Int $\mathrm{J}$ Tuberc Lung Dis 2003; 7: 607-08.

34. Lemus D, Martin A, Montoro E, Portaels F, Palomino JC. Rapid alternative methods for detection of rifampicin resistance in Mycobacterium tuberculosis. J Antimicrob Chemother 2004; 51: 130-33.

35. WHO. Molecular line probe assays for rapid screening of patients at risk of multidrug-resistant tuberculosis (MDR-TB). Policy statement. Geneva: World Health Organization, 2008.

36. Moore DA, Evans CA, Gilman RH. Microscopic-observation drug-susceptibility assay for the diagnosis of TB. N Eng J Med 2006; 355: 1539-50.

37. Singh S, Sankar MM, Gopinath K. High rate of extensively drug-resistant tuberculosis in Indian AIDS patients. AIDS 2007; 21: 2345-47. 
38. Kim H, Hwang SS, Kim HJ. Impact of extensive drug resistance on treatment outcomes in non-HIV-infected patients with multidrug-resistant tuberculosis. Clin Infect Dis 2007; 45: $1290-95$

39. Migliori GB, Ortmann J, Girardi E. Extensively drug-resistant tuberculosis, Italy and Germany. Emerg Infect Dis 2007; 13: 780-81.

40. Yew WW, Leung CC. Management of multidrug-resistant tuberculosis: Update 2007. Respirology 2008; 13: 21-46.

41. Dhingra VK, Rajpal S, Mittal A, Hanif M. Outcome of multidrug resistant tuberculosis cases treated by individualized regimens at a tertiary level clinic. Indian J Tuberc 2008; 55: 15-21.

42. Mukherjee JS, Rich ML, Socci AR. Programmes and principles in treatment of multidrug-resistant tuberculosis. Lancet 2004; 363: 474-81.

43. Rich ML, Socci AR, Mitnick CD, Nardell EA. Representative drug susceptibility patterns for guiding design of retreatment regimens for MDR-TB. Int J Tuberc Lung Dis 2006; 10: 290-96.

44. Han LL, Sloutsky A, Canales R, Naroditskaya V. Acquisition of drug resistance in multidrug-resistant Mycobacterium tuberculosis during directly observed empiric retreatment with standardized regimens. Int J Tuberc Lung Dis 2005; 9: 818-21.

45. Iseman M. A clinician's guide to tuberculosis. Philadelphia: Williams, and Wilkins, Lippincott, 2000.

46. Mitnick C, Bayona J, Palacios E. Community-based therapy for multidrug-resistant tuberculosis in Lima, Peru. N Engl J Med 2003; 348: 119-28.

47. WHO. Treatment of tuberculosis: guidelines for national programmes. WHO/CDS/TB/2003.313. Geneva: World Health Organization, 2003.
48. Iseman MD, Madsen L, Goble M, Pomerantz M. Surgical intervention in the treatment of pulmonary disease caused by drug-resistant Mycobacterium tuberculosis. Am Rev Respir Dis 1990; 141: 623-25.

49. Keshavjee S, Gelmanova I, Farmer P, Mishustin S, Strelis A, Andreev Y et al. Treatment of extensively drug-resistant tuberculosis in Tomsk, Russia: a retrospective cohort study. The Lancet 2008; 372: 1403-06.

50. Pomerantz BJ, Cleveland JC, Olson HK, Pomerantz M. Pulmonary resection for multidrug-resistant tuberculosis. J Thorac Cardiovasc Surg 2001; 121: 448-53.

51. Andries K, Verhasselt P, Guillemont J, et al. A diarylquinolone drug active on the ATP synthase of Mycobacterium tuberculosis. Science 2005; 307: 223-27.

52. Stover CK, Warrener P, Van Devanter DR. A small-molecule nitroimidazopyran drug candidate for the treatment of tuberculosis. Nature 2000; 405: 962-66.

53. Matsumoto M, Hashizume H, Tomishige T. OPC-6783, a nitro-dihydroimidazooxazole derivative with promising action against tuberculosis in vitro and in mice. PLoS Med 2006; 3: 466-70.

54. Nuermberger E, Tyagi S, Williams KN, Rosenthal I, Bishai WR, Grosset JH. Rifapentine, moxifloxacin, or DNA vaccine improves treatment of latent tuberculosis in a mouse model. Am J Respir Crit Care Med 2005; 11: 1452-56.

55. WHO. WHO Global Task Force outlines measures to combat XDR-TB worldwide. http://www.who.int/mediacentre/news/ notes/2006/np29/en/index.html. (Accessed Oct 7, 2008).

56. Currie CS, Williams BG, Cheng RC, Dye C. Tuberculosis epidemics driven by HIV: is prevention better than cure? AIDS 2003; 17: 2501-08. 